\title{
Effect of magnetic reconnection on CT penetration into magnetized plasmas
}

\author{
Yoshio Suzuki $^{1}$, Takaya Hayashi ${ }^{2}$, and Yasuaki Kishimoto ${ }^{1}$ \\ ${ }^{1}$ Naka Fusion Research Establishment, Japan Atomic Energy Research Institute, Ibaraki 311-0193, Japan \\ ${ }^{2}$ National Institute for Fusion Science, Gifu 509-5292, Japan
}

(Received May 30, 2000; Revised December 25, 2000; Accepted January 6, 2001)

\begin{abstract}
To understand the fuelling process in a fusion device by a compact toroid (CT) injection method, three dimensional MHD numerical simulations, where a spheromak-like CT (SCT) is injected into magnetized target plasmas, has been carried out. It has been found that the SCT penetration into magnetized target plasmas is accompanied by complex physical dynamics, which is not simply described by the conventional simple theoretical model. One of the most remarkable phenomena is magnetic reconnection. Magnetic reconnection plays a role in supplying the high density plasma, initially confined in the SCT magnetic field, to the target region. Furthermore, it is suggested that magnetic reconnection relaxes the deceleration of the SCT.
\end{abstract}

\section{Introduction}

In recent years, compact toroid (CT) plasmoid injection has been considered as one of the advanced fuelling methods for a fusion reactor, since the injection velocity of fuel is much faster than that by any of the other methods. It has been discussed in relation to several experimental (Brown et al., 1992; Raman et al., 1994; Raman et al., 1997; Raman et al., 1999; Gouge et al., 1997; Slough et al., 1996; Yee and Bellan, 1998; Fukumoto et al., 1996; Ogawa et al., 1999; Miyazawa et al., 1998) and theoretical (Parks et al., 1988; Perkins et al., 1988; Newcomb, 1991; Hwang et al., 1999) studies. Although these studies have shown the possibility of fuelling by this method, several unsolved problems have been remaining. We especially pay attention to the physical mechanism to determine the CT penetration depth and carry out three dimensional MHD numerical simulations, where a spheromak-like CT (SCT) is injected into the magnetized target plasma region.

In our previous paper (Suzuki et al., 2000), it is revealed that 1) the magnetic configuration of the injected SCT is disrupted by magnetic reconnection between the SCT magnetic field and the target magnetic field, which leads to supply of the SCT high density plasma, 2) the penetration depth of the SCT plasma into the target region becomes shorter than that estimated from the conducting sphere (CS) model, because the Lorentz force of the target magnetic field sequentially decelerates the SCT through the plasma compressibility, while in the CS model only the magnetic pressure force decelerates it. In this study, we present a new theoretical model to determine the SCT penetration depth and investigate in detail the deceleration mechanism of the injected SCT by comparison with simulation results.

Copy right $(\mathrm{c}$ The Society of Geomagnetism and Earth, Planetary and Space Sciences (SGEPSS); The Seismological Society of Japan; The Volcanological Society of Japan; The Geodetic Society of Japan; The Japanese Society for Planetary Sciences.

\section{Theoretical Model}

So far, the CS model has been widely employed to estimate the penetration depth of the injected CT (Raman et al., 1994; Gouge et al. 1997; Ogawa et al, 1999). This model indicates that the CT, for which an incompressible, perfectly conducting sphere is substituted, penetrates by slipping through the target magnetic field, that is, the CT can penetrate until its initial kinetic energy is exceeded by the magnetic energy required to exclude the magnetic field from its volume:

$$
\frac{d}{d t} \int_{\mathrm{CT}} \frac{1}{2} \rho V^{2} d v=-\frac{d}{d t} \int_{\mathrm{CT}} \frac{1}{2 \mu_{0}} B^{2} d v
$$

where $\rho, V$ and $B$ are the CT density, the CT velocity and the strength of the target magnetic field at the point where the CT is penetrating, respectively. Taking $V=V_{\mathrm{CT}}$ and $B=0$ for the initial condition, and $V=0$ and $B=B_{\text {target }}$ for the final condition when the CT stops, we can lead

$$
\frac{1}{2} \rho V_{\mathrm{CT}}^{2} v_{\mathrm{CT}}=\frac{1}{2 \mu_{0}} B_{\text {target }}^{2} v_{\mathrm{CT}}
$$

where $v_{\mathrm{CT}}$ is the $\mathrm{CT}$ volume. Thus, the $\mathrm{CT}$ can penetrate until the point where the strength of the target magnetic field is

$$
B_{\text {target }}=\sqrt{\mu_{0} \rho} V_{\mathrm{CT}} .
$$

Furthermore, taking a $z$ axis for the injection direction, we can lead from Eq. (1) the equation of motion as follows:

$$
\begin{aligned}
\frac{d}{d t} \int_{\mathrm{CT}} \frac{1}{2} \rho V_{z}^{2} d v & =\int_{\mathrm{CT}}-\frac{\partial z}{\partial t} \frac{\partial}{\partial z} \frac{1}{2 \mu_{0}} B^{2} d v \\
\int_{\mathrm{CT}} V_{z} \frac{\partial}{\partial t} \rho V_{z} d v & =\int_{\mathrm{CT}}-V_{z} \frac{\partial}{\partial z} \frac{1}{2 \mu_{0}} B^{2} d v \\
\frac{\partial}{\partial t} \rho V_{z} & =F-\frac{\partial}{\partial z} \frac{B^{2}}{2 \mu_{0}}
\end{aligned}
$$

where $F$ is the CT acceleration force. Therefore, in this model only the magnetic pressure force acts as the deceleration mechanism of the CT. However, the CT actually has the 


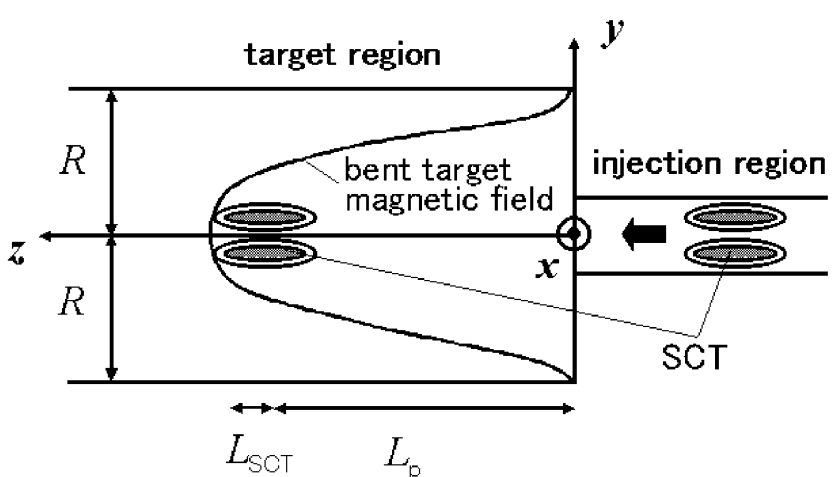

Fig. 1. Schematic diagram of the bent target magnetic field.

finite resistivity, which could inhibit the CT from slipping through the target magnetic field. From the simulation with finite resistivity, it has been confirmed that the injected SCT cannot slip the target magnetic field but bent it in the SCT penetration direction. In addition, it has been revealed that the Lorentz force of the target magnetic field sequentially decelerates the SCT through the penetration process (Suzuki et al., 2000). The Lorentz force consists of the magnetic pressure force and the magnetic tension force:

$$
\boldsymbol{J} \times \boldsymbol{B}=-\nabla\left(B^{2} /\left(2 \mu_{0}\right)\right)+(\boldsymbol{B} \cdot \nabla) \boldsymbol{B} / \mu_{0} .
$$

Therefore, the magnetic tension force could also decelerate the SCT. If we approximate that the target magnetic field is bent with a cosine function as shown in Fig. 1, the magnetic tension force is lead as follows:

$$
\begin{aligned}
& z=\frac{L_{p}+L_{\mathrm{SCT}}}{2} \cos \left(\frac{\pi y}{R}\right)+\frac{L_{p}+L_{\mathrm{SCT}}}{2} \\
& \frac{d z}{d y}=\frac{B_{z}}{B_{y}}=-\frac{\pi\left(L_{p}+L_{\mathrm{SCT}}\right)}{2 R} \sin \left(\frac{\pi y}{R}\right) \\
& \boldsymbol{B}=B\left[-\frac{\pi\left(L_{p}+L_{\mathrm{SCT}}\right)}{2 R} \sin \left(\frac{\pi y}{R}\right) \boldsymbol{z}+\boldsymbol{y}\right] \\
& {\left[\boldsymbol{B} \cdot \nabla B_{z} / \mu_{0}\right]_{y=0}=-\frac{B^{2}}{\mu_{0}} \frac{\pi^{2}\left(L_{p}+L_{\mathrm{SCT}}\right)}{2 R^{2}}}
\end{aligned}
$$

where $L_{\mathrm{SCT}}, L_{p}$ and $R$ are the half size of the SCT, the SCT penetration depth and the radius of the target region, respectively. Thus, the equation of motion for the SCT is given by adding it to Eq. (6):

$$
\frac{\partial}{\partial t} \rho V_{z}=F-\frac{\partial}{\partial z} \frac{B^{2}}{2 \mu_{0}}-\frac{B^{2} \pi^{2}\left(L_{p}+L_{\mathrm{SCT}}\right)}{2 \mu_{0} R^{2}} .
$$

Neglecting the convection term, we call it the nonslipping sphere (NS) model. Thus, we respectively represent,

CS model:

$$
\rho \frac{\partial V_{z}}{\partial t}=F-\frac{\partial}{\partial z} \frac{B^{2}}{2 \mu_{0}}
$$

NS model:

$$
\rho \frac{\partial V_{z}}{\partial t}=F-\frac{\partial}{\partial z} \frac{B^{2}}{2 \mu_{0}}-\frac{B^{2} \pi^{2}\left(L_{p}+L_{\mathrm{SCT}}\right)}{2 \mu_{0} R^{2}}
$$

where the magnetic pressure force and the magnetic tension force are averaged over the SCT volume. Furthermore, from

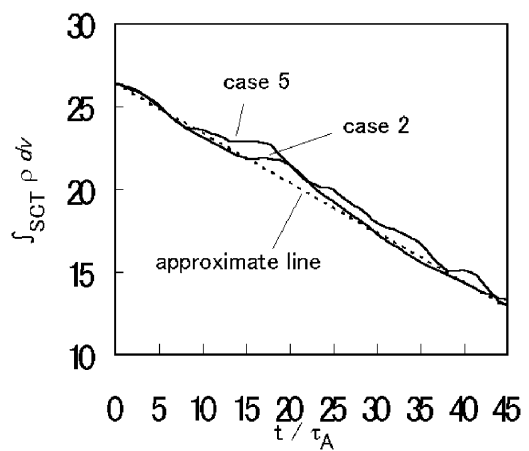

Fig. 2. Time evolution of the SCT (the bare plasmoid) mass. The broken line is approximated by the equation: $-0.3 t+\rho_{\text {init }} v_{\text {init }}$.

the simulation result, we approximate the decrease of the SCT density with the following equation (approximate line in Fig. 2),

$$
\rho=-\frac{0.3}{v_{\text {init }}} t+\rho_{\text {init }}
$$

where $\rho_{\text {init }}$ and $v_{\text {init }}$ are the initial SCT density and the initial SCT volume, respectively. In each model, the SCT penetration depth is determined by simultaneously solving

$$
\frac{d L_{p}}{d t}=V_{z}
$$

To examine clearly the effect of the magnetic pressure force and the magnetic tension force, $F$ is estimated from cases with null target magnetic field $\left(B_{\text {target }}=0\right)$.

\section{Simulation Model}

The simulation model is the same as in our previous paper (Suzuki et al., 2000). The simulation region is composed of two cylinders which connect with each other (Fig. 3). One with a smaller radius $L_{r}$ corresponds to the SCT gun (injection region) and the other with a larger radius $4 L_{r}$ corresponds to a part of a fusion device (target region). Lengths of these cylinders are both $L_{z} / 2$. The relative lengths of the sizes are given by $L_{r}: L_{z}=1: 16$. We employ a perfectly conducting wall for the boundary condition.

The governing equations are given by MHD equations:

$$
\begin{aligned}
\frac{\partial \rho}{\partial t}= & -\nabla \cdot(\rho \boldsymbol{V}), \\
\frac{\partial \rho \boldsymbol{V}}{\partial t}= & -\nabla \cdot \rho \boldsymbol{V} \boldsymbol{V}+\boldsymbol{J} \times \boldsymbol{B}-\nabla P-\nabla \cdot \Pi, \\
\frac{\partial \boldsymbol{B}}{\partial t}= & -\nabla \times \boldsymbol{E}, \\
\frac{\partial P}{\partial t}= & -\nabla \cdot(P \boldsymbol{V}-\kappa \nabla T) \\
& -(\gamma-1)\left(P \nabla \cdot \boldsymbol{V}+\Pi: \nabla \boldsymbol{V}-\eta J^{2}\right), \\
\boldsymbol{E}= & -\boldsymbol{V} \times \boldsymbol{B}+\eta \boldsymbol{J}, \\
\boldsymbol{J}= & \nabla \times \boldsymbol{B}, \\
\boldsymbol{J}= & \nabla \times \boldsymbol{B}, \\
\Pi= & P / \rho, \\
\Pi= & \nu\left[\frac{2}{3}(\nabla \cdot \boldsymbol{V}) \mathbf{I}-\nabla \boldsymbol{V}-{ }^{t}(\nabla \boldsymbol{V})\right] .
\end{aligned}
$$


Table 1. Several parameters in nine different simulation runs. Cases 1, 2, 4 and 5 correspond to those in the previous simulation. The SCT magnetic field and the SCT density are shown by their averages and their maximum values are 1 and 10 in the normalized unit, respectively.

\begin{tabular}{cccccccc}
\hline & \multicolumn{3}{c}{$\mathrm{SCT}$} & \multicolumn{3}{c}{ Target } & \multicolumn{2}{c}{ Common } \\
Case & $V_{\mathrm{SCT}}$ & $\left\langle\rho_{\mathrm{SCT}}\right\rangle$ & $\left\langle B_{\mathrm{SCT}}\right\rangle$ & $\rho_{\text {target }}$ & $B_{\text {target }}$ & $P_{\text {com }}$ & $\eta$ \\
\hline 1 & 0.3 & 5.5 & 0.46 & 0.1 & 0 & 0.1 & $1.0 \times 10^{-3}$ \\
2 & 0.3 & 5.5 & 0.46 & 0.1 & 0.1 & 0.1 & $1.0 \times 10^{-3}$ \\
3 & 0.3 & 5.5 & 0.46 & 0.1 & 0.3 & 0.1 & $1.0 \times 10^{-3}$ \\
4 & 0.3 & 5.5 & 0 & 0.1 & 0 & 0.1 & $1.0 \times 10^{-3}$ \\
5 & 0.3 & 5.5 & 0 & 0.1 & 0.1 & 0.1 & $1.0 \times 10^{-3}$ \\
6 & 0.3 & 5.5 & 0.46 & 0.1 & 0 & 0.1 & $5.0 \times 10^{-4}$ \\
7 & 0.3 & 5.5 & 0.46 & 0.1 & 0.1 & 0.1 & $5.0 \times 10^{-4}$ \\
8 & 0.3 & 5.5 & 0.46 & 0.1 & 0 & 0.1 & $2.0 \times 10^{-4}$ \\
9 & 0.3 & 5.5 & 0.46 & 0.1 & 0.1 & 0.1 & $2.0 \times 10^{-4}$ \\
\hline
\end{tabular}

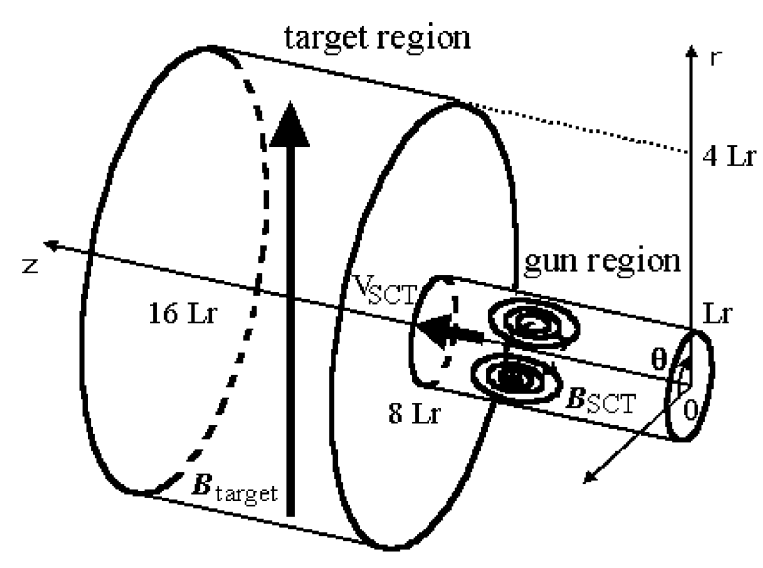

Fig. 3. Schematic diagram of the simulation region.

These equations have a non-dimensional form, in which the density, the magnetic field, the velocity, the length and the time are normalized by one-tenth of the maximum SCT density $\rho_{0}\left(\equiv 0.1 \rho_{\mathrm{SCT}}\right)$, the maximum strength of the SCT magnetic field $B_{0}\left(\equiv B_{\mathrm{SCT}}\right)$, the characteristic Alfvén velocity $V_{A}\left(\equiv B_{0} / \sqrt{\mu_{0} \rho_{0}}\right)$, the scale of the cylinder radius $L_{r}$, and the Alfvén transit time $\tau_{A}\left(\equiv L_{r} / V_{A}\right)$, respectively. The resistivity $\eta$, the viscosity $v$ and the conductivity $\kappa$ are fixed to $1.0 \times 10^{-3}$ in the normalized unit $\mu_{0} L_{r} V_{A}, \rho_{0} L_{r} V_{A}$ and $k \rho_{0} L_{r} V_{A}$, respectively.

The initial magnetic configuration is calculated as follows: The toroidal current $J_{\theta}$ of a cylindrical spheromak solution (Finn and Manheimer, 1981) whose radius and length are respectively $L_{r}$ and $2 L_{r}$ is located in the injection region. From $J_{\theta}$, the toroidal component of the vector potential $A_{\theta}$, and thus the magnetic field is calculated. Furthermore, the current free target magnetic field $B_{\text {target }}$, whose strength is an almost uniform and direction is perpendicular to the injection direction is superposed. The density profile is given by the function of the SCT poloidal flux. The target plasma density $\rho_{\text {target }}$ is assumed to be one percent of the peak SCT plasma density $\rho_{\mathrm{SCT}}$. The pressure is initially uniform $\left(P_{\text {com }}=P_{\text {target }}=P_{\mathrm{SCT}}\right)$. The SCT is injected into the target region after acceleration up to the maximum velocity $V_{\mathrm{SCT}}$ at

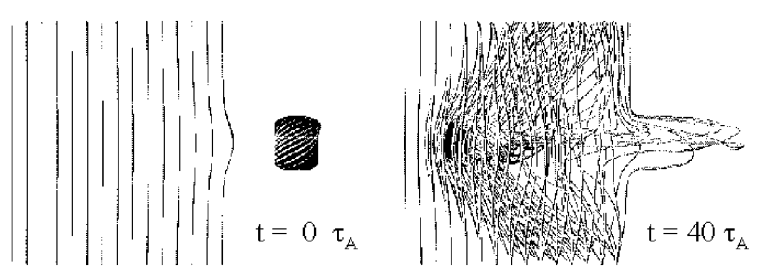

Fig. 4. The spatial structure of the SCT high density plasma, the SCT magnetic field, and the target magnetic field in case 2 .

$t=t_{\mathrm{off}} \equiv 2 L_{r} / V_{\mathrm{SCT}}$. The acceleration is done for $\rho>\rho_{\mathrm{c}}$.

The explicit finite difference method with second-order accuracy and the Runge-Kutta-Gill method are used to solve the basic equations numerically. We carry out totally nine simulation runs as shown in Table 1 . Cases 1, 2, 4 and 5 are the same as previous ones.

In the next section, we compare the penetration depth and the kinetic energy of the SCT between models and simulation. In the NS model, $L_{\mathrm{SCT}}$ and $R$ are given by $L_{r}$ and $4 L_{r}$, respectively.

\section{Comparison of the Model with the Simulation Results}

Figure 4 shows the spatial structure of the SCT high density plasma and magnetic field lines at $t=0$ and $40 \tau_{A}$ in case 2. At $t=0 \tau_{A}$, the SCT is located in the gun region and the target magnetic field is almost uniform. At $t=40 \tau_{A}$, the SCT penetrates the target region with bending the taget magnetic field in the SCT penetration direction. In addition, as a result of magnetic reconnection between the SCT magnetic field and the target magnetic field, the SCT magnetic configuration is disrupted, which leads to supply of the SCT high density plasma in the target region. The detailed structure of reconnected field lines is shown in our previous paper (Suzuki et al., 2000).

Figure 5 shows the SCT penetration depth given by the CS model, the NS model and the simulation (case 2). It can be seen that the SCT penetration depth in the NS model well coincides with that in the simulation, while it in the CS model is longer. Thus, the magnetic tension force also effectively 


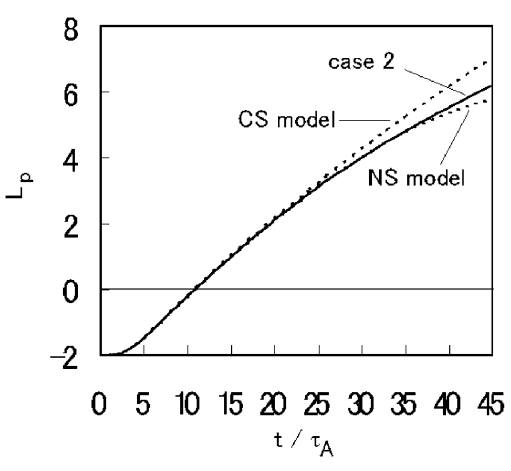

Fig. 5. Time evolution of SCT penetration depths in the CS model, the NS model and case 2 . The target region corresponds to $0<L_{p}<8 L_{r}$.

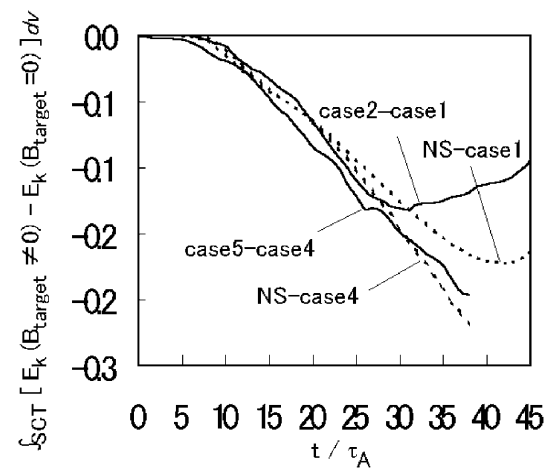

Fig. 6. Time evolution of SCT kinetic energy in the NS model and cases 2 and 5 , which is shown by subtracting the corresponding null target magnetic field case.

acts as the deceleration mechanism of the SCT. However, the SCT penetration depth in the NS model is a little shorter than that in the simulation with time. Figure 6 shows the time evolution of SCT kinetic energy in the NS model and case 2 by subtracting the corresponding null target magnetic field case from them. It can be seen that the SCT kinetic energy in the NS model well coincides with that in the simulation until about $30 \tau_{A}$. After that, however, the SCT kinetic energy in the simulation begins to increase, while it in the NS model continues to decrease. It causes the longer penetration depth of the SCT than that estimated from the NS model.

Now, let us examine the effect of magnetic reconnection, which could relax the SCT deceleration, since it can dynamically change the topology of the magnetic field. Here, we use the simulation result (case 5), in which the bare plasmoid, that is, the SCT with null magnetic field is injected. All parameters except the SCT magnetic field in this case are the same as those in case 2. Since it is assumed that the initial pressure profile is spatially constant and the heat conductive time is longer than the Alfvén time, the diffusion of the high density plasma is not so different from that in the SCT injection (Fig. 2). In addition, it is obvious that magnetic reconnection observed in case 2 does not occur. In Fig. 6, the time evolution of kinetic energy in case 5 is also shown. It is found that this time evolution coincides with that in the NS model more than that of the SCT kinetic

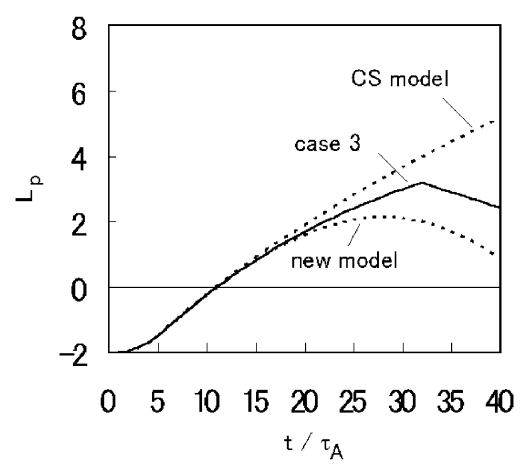

Fig. 7. Time evolution of SCT penetration depths in the CS model, the NS model and case 3 .

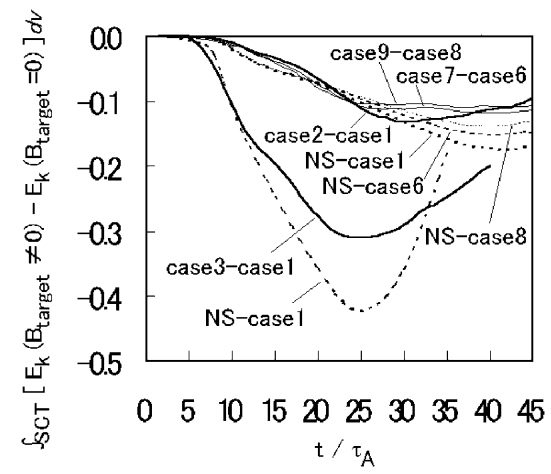

Fig. 8. Time evolution of SCT kinetic energy in the NS model and cases 2, 3,7 , and 9 .

energy in case 2 does. These results suggest that magnetic reconnection relaxes the SCT deceleration. The reconnection process could give the SCT penetration the same effect as the slipping indicated in the CS model.

\section{Parameter Dependence}

Figure 7 shows the SCT penetration depth in case 3. It is found that in this case also the SCT penetration depth in the CS model is longer than that in the simulation. On the other hand, it in the NS model becomes shorter. Figure 8 shows the time evolution of SCT kinetic energy in this case. The difference of the SCT kinetic energy between the NS model and the simulation is larger when the target magnetic field is stronger, which causes the larger difference of the SCT penetration depth between them.

In Figure 8, the time evolution of SCT kinetic energy in cases with different resistivity (cases 7 and 9) is also shown. These results show that the dependence of the resistivity is not so large. Since there are more than ten grid numbers within a current sheet around the reconnection point, the effect of the numerical diffusion is small enough. The detailed relation between the relaxation time scale and the reconnection rate has not been understood yet.

\section{Summary}

We investigate the deceleration mechanism of the SCT injected into the magnetized target plasma region. It is revealed that in the SCT penetration process both the magnetic 
pressure force and the magnetic tension force effectively decelerate the SCT. In addition, it is suggested that magnetic reconnection relaxes the SCT deceleration. In the current simulation, however, the both ends of the target magnetic field is fixed on the boundary wall, which would cause the overestimate of the magnetic tension force. This dependence will be reported in our future work.

\section{References}

Brown, M. R. and P. M. Bellan, Efficiency and scaling of current drive and refueling by sheromak injection into a tokamak, Nucl. Fusion, 32, 1125-1137, 1992.

Finn, J. M. and W. M. Manheimer, Spheromak tilting instability in cylindrical geometry, Phys. Fluids, 24, 1336-1341, 1981.

Fukumoto, N., et al., Compant torus acceleration and injection experiment, Bull. Am. Phys. Soc., 41, 1578, 1996.

Gouge, M. J., et al., Development of innovative fuelling systems for fusion energy science, Fusion Energy 1996, Proc. 16th Int. Conf. Montreal, 1996, Vol. 3, IAEA, Vienna, 481, 1997.

Hwang, D. Q., M. Ryutova, and H. McLean, Penetration of a compressible magnetized plasma object into a low beta taret plasma, Phys. Plasmas, 6, 1515-1521, 1999.

Miyazawa, J., H. Yamada, and O. Motojima, Possibility of profile control using compact toroid injection on Large Helical Devide, Jpn. J. Appl. Phys., 37, 6620-6627, 1998.
Newcomb, W. A., Magnetohydrodynamic wave drag, Phys. Fluids B, 3, 1818-1829, 1991.

Ogawa, T., et al., Compact toroid injection experiment in JFT-2M, Nucl. Fusion, 39, 1911-1915, 1999.

Parks, P. B., Refuelling tokamaks by injection of compact toroids, Phys. Rev. Lett., 61, 1364-1367, 1988.

Perkins, L. J., S. K. Ho, and J. H. Hammer, Deep penetration fuelling of reactor-grade tokamak plasmas with accelerated compact toroids, $\mathrm{Nucl}$. Fusion, 28, 1365-1378, 1988.

Raman, R., et al., Experimental demonstration of nondisruptive, central fueling of a tokamak by compact toroid injection, Phys. Rev. Lett., 73, 3101-3104, 1994.

Raman, R., et al., Experimental demonstration of tokamak fuelling by compact toroid injection, Nucl. Fusion, 37, 967-972, 1997.

Raman, R., et al., CT fuelling of Tdev, Controlled Fusion and Plasma Physics, Proc. 24th Eur. Conf. Berchtesgaden, 1997, Vol. 21A, Part I, European Physical Society, Geneva, 293, 1999.

Slough, J. T. and A. L. Hoffman, Acceleration of a field reversed configuration for central fueling of ITER, Fusion Energy 1996, Proc. 16th Int. Conf. Montreal, 1996, Vol. 2, IAEA, Vienna, 237-242, 1997.

Suzuki, Y., et al., Three dimensional simulation study of spheromak injection into magnetized plasmas, Nucl. Fusion, 40, 277-288, 2000.

Yee, J. and P. M. Bellan, Effects of CT injector acceleration electrode configuration on tokamak penetration, Nucl. Fusion, 38, 711-721, 1998.

Y. Suzuki (e-mail: suzukiy @ fusion.naka.jaeri.go.jp), T. Hayashi, and Y. Kishimoto 